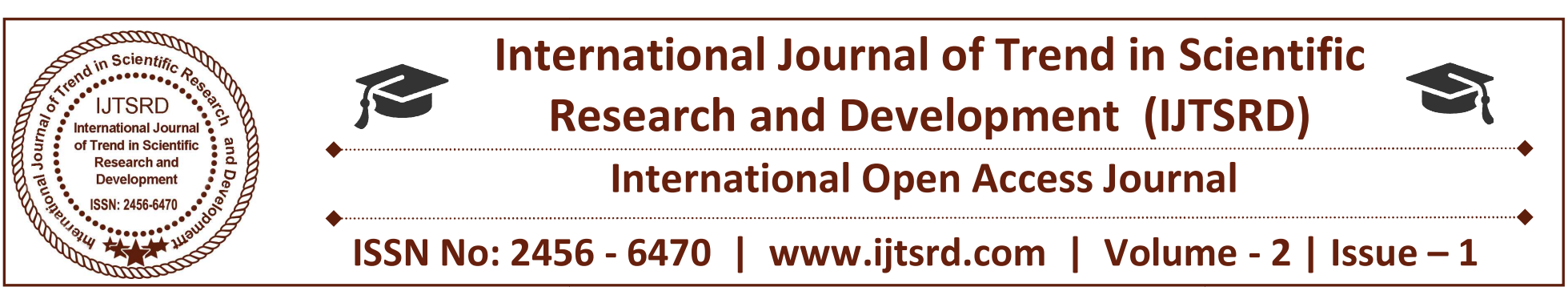

\title{
Radiation effects on MHD Boundary Layer Flow Over A Moving Vertical Porous Plate with Heat Generation in the Presence of Chemically Reacting Non Newtonianfluid and Soret Effect
}

\author{
G Vidyasagar \\ Assistant Professor, Department of Basic Sciences \\ and Humanities, QIS College of Engineering and \\ Technology, Ongole, Prakasam District, A.P., India
}

\begin{abstract}
B Ramana
Assistant Professor, Department of Basic Sciences and Humanities, QIS College of Engineering and Technology, Ongole, Prakasam District, A.P., India
\end{abstract}

\section{ABSTRACT}

This paper is focused on the study of effect of heat and mass transfer on chemically reacting boundary layer flow of a Casson fluid over a porous stretching sheet in the differential equations are transformed by introducing similarity variables and solved numerically by using Range Kutta method along with shooting method. The velocity, temperature and concentration distributions are discussed number tables for different parameters entering into the problem. It is observed that the Casson parameter decreases the velocity field while the temperature is enhanced with increasing Casson parameter.

Keywords: Mass Transfer, MHD, Casson Parameter, suction, heat generation, chemical reaction, radiation effect and Soret effect

\section{Introduction:}

The combined effects of heat and mass transfer with chemical reaction have attracted many researchers due to its wide range of applications in Engineering and Science. Heat and mass transfer occur simultaneously in the processes of drying and evaporation at the surface of a water body, energy transfer in a wet cooling tower and the flow in a desert cooler. The study of magnetohydrodynamic (MHD) flow of an electrically conducting fluid is of considerable interest due to its application in many engineering problems such as MHD generators, plasma studies, nuclear reactors, and geothermal energy extractions. Das et al. (1994) studied the first order chemical reaction effect on the flow past

an impulsively started infinite vertical plate with constant heat flux and mass transfer. Anjalidevi and Kandaswamy (1999) considered the heat and mass transfer on steady laminar flow along a semi-infinite horizontal plate in the presence of chemical reaction. The Soret and Dufour effects on heat and mass transfer about vertical surfaces in porous media have been studied by Postelnicu (2004). radiation on boundary layer flow over a moving vertical porous plate was analyzed by Makinde (2005). Muthucumaraswamy et al. (2006) have studied the effect of chemical reaction isothermal vertical plate with radiation. Mandal (1985)investigated mass transfer effects on unsteady hydromagnetic free convection flow past an accelerated vertical porous plate. Jha (1991) studied the effect of magnetic field on mass transfer flow past a uniformly accelerated vertical plate through a porous medium. Elbashbeshy (1997) analyzed the heat and mass transfer along a vertical plate in the presence of magnetic field. The combined heat and mass transfer convection flow from a vertical surface with Ohmic heating and viscous dissipation was analyzed by Chen (2004).

The study of boundary layer flow over a stretching sheet has finds applications in chemical engineering, particularly in manufacture artificial film, artificial fibers, polymer extrusion, drawing of plastic films and wires, glass fiber and paper production. Crane (1970) was the first researcher who investigated the boundary layer flow over a stretching surface. After the study of fluid flow over a stretching sheet has received wide 
attention among researchers. Rajagopal et al. (1984) discussed the flow of second order fluid over a stretched sheet. Anderson et al. (1992) consider magnetic field on the flow of a viscoelastic fluid past a stretching sheet. Abel et al. (2005) analyzed MHD boundary layer flow over continuously moving stretching surface embedded in a porous medium by considering the Buoyancy force and effects. Mukhopadyaya et al. (2008) discussed the free convective boundary layer flow with variable viscosity over a stretching surface with thermal radiation. Pal (2009) investigated the mixed convection flow of an incompressible fluid over a stretching sheet in the presence of radiation. Ahmed (2009) analyzed the free convective heat and mass transfer of a viscous incompressible fluid over a stretching sheet in the presence of suction with Soret and Dufour effects. Heat and mass transfer in non-Newtonian fluids have applications in engineering such as catalytic reactors, the filtration and blood plasmapheresis devices. Casson fluid is the most popular non-Newtonian fluid used to model blood. It is defined as a shear thinning liquid which has a infinite viscosity at zero rate of shear, possessing a yield stress below which no flow occurs and zero viscosity at infinite rate of shear. It is reduced to Newtonian fluid at very high wall shear stress i.e. when the wall stress is much greater than yield stress. Merill et al. (1965) and McDonald (1974) conducted experiments on the behavior of blood as a Casson fluid. Eldabe (1995) considered the heat transfer of Casson fluid flow between two rotating cylinders. The flow of Casson fluid in a tube was studied by Dash et al. (2000) and Nagarani et al. (2004). Mass transfer in a Casson flowing through an annular geometry was examined by Nagarani et al. (2006). Attia (2010) analyzed the transient Couette flow of a Casson fluid between parallel plates with magnetic field and heat transfer. The unsteady boundary layer flow of a Casson fluid over a moving flat plate was studied by Mustafa et al. (2011). Hayat et al. (2012) studied the mixed convection stagnation point flow of a Casson fluid. Shehzad (2013) discussed the effects of mass transfer on the MHD boundary layer flow of a Casson fluid with chemical reaction.

In view of the above studies, we consider the MHD boundary layer flow of a Casson fluid over a stretching sheet with heat and mass transfer and chemical reaction.

\section{.Nomenclature:}

$\mathrm{u}, \mathrm{v} \quad$ : Velocity components along the $\mathrm{x}$ and $\mathrm{y}$ axes

$\mathrm{T}$ : $\quad$ Fluid temperature inside the boundary layer

$T_{\infty} \quad$ : $\quad$ Ambient temperature for away from the plate

$T_{w} \quad: \quad$ Uniform constant temperature at the wall

C : Species concentration inside the boundary layer

$C_{\infty} \quad: \quad$ Species concentration of the ambient fluid

g : : Acceleration due to gravity

$k^{\prime} \quad$ : $\quad$ Permeability of the porous medium

$B_{0} \quad$ : $\quad$ Uniform magnetic field

Pr : Prandtl number

M : Magnetic parameter

Gr : $\quad$ Grashof number

Gc : $\quad$ Modified Grashof number

Sc : $\quad$ Schmidtnumber

D : Molecular diffusivity of the species concentration

K : : Permeability parameter

\section{Greek Symbols:}

$\theta$ : Dimensionless temperature

$\rho$ : $\quad$ Fluid density

ᄂ : Kinematics viscosity

$\sigma: \quad$ Electrical conductivity

$\beta, \beta^{*}: \quad$ Thermal and concentration expansion coefficient

$\alpha \quad: \quad$ Thermal diffusivity

$F_{w} \quad: \quad$ Suction parameter 


\section{Mathematical Formulation:}

Consider the steady, incompressible flow of a Casson fluid over a porous stretching surface at $y=0$. Choose the coordinate system such that $\mathrm{x}$-axis is parallel to the surface and $y$-axis normal to the surface. The fluid occupies half space $\mathrm{y}>0$. A uniform magnetic field B0 is applied in the $\mathrm{y}$ direction. The transverse applied magnetic field and magnetic Reynolds number are assumed to be very small, so that the induced magnetic field and Hall effects are negligible. We also considered the heat and mass transfer processes in the presence of chemical reaction and suction. The rheological equation of state for an isotropic and incompressible flow of a Casson fluid can be written as (Nakamura and Sawada (1988), Mustafa et al. (2012)).

$$
\tau_{i j}=\begin{aligned}
& 2\left(\mu_{B}+P_{y} / \sqrt{2 \pi}\right) e_{i j}, \pi>\pi_{c} \\
& 2\left(\mu_{B}+P_{y} / \sqrt{2 \pi}\right) e_{i j}, \pi<\pi_{c}
\end{aligned}
$$

Where $\mu \mathrm{B}$ is the plastic dynamic viscosity of the nonNewtonian fluid, Py is the yield stress of the fluid, e ij denotes the $(i, j)$ - th component of the deformation rate, $\pi=\mathrm{e} i \mathrm{j}$ e $\mathrm{ij}$ is the product of the component of deformation rate with itself, $\pi \mathrm{c}$ is the critical value of $\pi$ based on the non-Newtonian model.

Under above conditions the continuity, momentum and energy equations are

Continuity Equation:

$$
\frac{\partial u}{\partial x}+\frac{\partial v}{\partial y}=0
$$

Momentum Equation:

$$
\begin{aligned}
& u \frac{\partial u}{\partial x}+v \frac{\partial u}{\partial y}=\gamma\left(1+\frac{1}{\beta}\right) \frac{\partial^{2} u}{\partial y^{2}}-\sigma \\
& \frac{B_{0}^{2}}{\rho} u-\frac{\gamma}{K^{*}} u+g \beta\left(T-T_{\infty}\right)+g \beta^{*}\left(C-C_{\infty}\right)
\end{aligned}
$$

Energy Equation:

$u \frac{\partial T}{\partial x}+v \frac{\partial T}{\partial y}=\alpha \frac{\partial^{2} T}{\partial y^{2}}-\frac{1}{\rho C_{p}} \frac{\partial q_{r}}{\partial y}+\frac{Q_{0}}{\rho C_{P}}\left(T-T_{\infty}\right)$
Concentration Equation:

$u \frac{\partial C}{\partial x}+v \frac{\partial C}{\partial y}=D \frac{\partial^{2} C}{\partial y^{2}}-k_{1}\left(C-C_{\infty}\right)+D_{1} \frac{\partial^{2} T}{\partial y^{2}}$

$\frac{\partial q_{r}}{\partial y}=4 \alpha^{2}\left(T-T_{\infty}\right)$

Where $\mathrm{u}$ and $\mathrm{v}$ are the velocity components in $\mathrm{x}$ and $\mathrm{y}$ directions, $\quad \gamma$ is the kinematic viscosity, $\beta=\mu_{B} \sqrt{2 \pi_{c}} / P_{Y}$ is the Casson fluid parameter, $\sigma$ is the electric Conductivity of the fluid, $\rho$ is the density of the fluid, $\mathrm{T}$ is the temperature of the fluid, $\mathrm{C}$ is the Concentration field, $\alpha$ is the thermal diffusivity, $\mathrm{D}$ is the mass diffusivity, $\mathrm{k}^{1}$ is the reaction rate.

The Boundary Conditions for the Velocity, temperature and Concentration fields are

$\mathrm{u}=\mathrm{u}^{w}, \mathrm{v}=-\mathrm{V}^{0}, T=T_{w}, C=C_{W} \quad$ when $\mathrm{y}=0$

$\mathrm{u} \rightarrow \mathrm{u}^{w}, \mathrm{~T} \rightarrow \mathrm{T} \infty, \mathrm{C} \rightarrow \mathrm{C} \infty$ as $\mathrm{y} \rightarrow \infty$

We define the following similarity variables as

$$
\begin{aligned}
\eta & =\sqrt{\frac{c}{\gamma}} y, f(\eta)=\frac{\psi}{x \sqrt{c \gamma}}, \theta(\eta)=\frac{T-T_{\infty}}{T_{w}-T_{\infty}}, \phi(\eta)=\frac{C-C_{\infty}}{C_{W}-C_{\infty}} \\
B & =\frac{Q_{0}}{C \rho C_{P}}
\end{aligned}
$$

Where $\Psi$ is the stream function with $\mathrm{u}=$ $\frac{\partial \psi}{\partial y}, v=-\frac{\partial \psi}{\partial x}$

$\eta_{\text {is }}$ the similarity variable substituting the nondimensional variables

In view of (2), (3) and (4) the Equations (2) to (4) take the form

$$
\begin{aligned}
& \left(1+\frac{1}{\beta}\right) f \\
& +f f^{\prime \prime}-\left(f^{\prime}\right)^{2}-(M+K) f^{\prime}=-(G r \theta+G c \phi) \\
& \frac{1}{\operatorname{Pr}} \theta^{\prime \prime}+f \theta^{\prime}-(R a-B) \theta=0
\end{aligned}
$$


$\phi^{\prime \prime}-\operatorname{Scf} \theta^{\prime}-S_{c} K_{1} \phi+\theta^{\prime \prime} S r S c=0$

where the primes denote the differentiation with respect

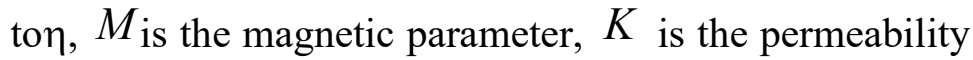
parameter, $G r$ is the temperature Grashof number, $G c$ is the concentration Grashof number, $\operatorname{Pr}$ is the Prandtl number, $\mathrm{Ra}$ is the Radiation parameter, $\mathrm{B}$ is the Heat Generation, $\mathrm{Sr}$ is the Soret effect and $S c$ is the Schmidt number.

The Corresponding non -dimensional boundary conditions are

$$
\begin{aligned}
& f^{\prime}=1, f=S, \theta=1, \phi=1, \text { when } \eta=0 \\
& f^{\prime}(\infty) \rightarrow 0, \theta(\infty) \rightarrow 0, \phi(\propto) \rightarrow 0 \text { as } \mathrm{y} \rightarrow \propto
\end{aligned}
$$

\section{Solution of the Problem}

The governing boundary layer equations (7) to (9) subject to boundary conditions (10) are solved numerically by using shooting method. First of all, higher order non-linear differential equations (7) to (9) are converted into simultaneous linear differential equations of first order and they are further transformed into initial value problem by applying the Range Kutta method along with shooting technique. From the process of numerical computation, the skin-friction coefficient, the Nusselt number and Sherwood number which are respectively proportional to $F(0),-\theta(0)$ and $-\phi(0)$ are also sorted out and their numerical values are presented in a tabular form.

\section{Results and Discussion}

In order to get a physical insight into the problem, a representative set of numerical results is shown graphically in Figs.1-27, to illustrate the influence of physical parameters viz., the effect of the Casson parameter $(\beta)$, Magnetic field $(\mathrm{M})$, Porous parameter $(\mathrm{K})$, Grashof Number (Gr), Modified Grashof number (Gc), Suction parameter (S), Prandtl Number (Pr), Radiation parameter (R), Heat Generation (B), Schmidt's Number (Sc), Chemical Reaction (K1) and Soret effect (Sr) on the flow variables have been graphically presented.

Fig 1 presents the effect of Casson parameter $(\beta)$ on velocity. It is observed that the velocity increases asymptotically from its highest value on the surfaces to zero as $\eta \rightarrow \infty$. The presence of yield stress reduces the velocity. Increasing values of $\beta$ increases the velocity further and thus there is a decrease in the thickness of the boundary layer.

Fig. 2 shows the variation of chemical reaction $(\mathrm{Ch})$ on velocity. The effect of chemical reaction increases with the increases on velocity. Fig 3 illustrates the effect of Grashof number (Gr) on velocity. We observe that the velocity decreases with the increase of Grashof number (Gr) with take the reverse action. Fig 4 illustrates the effect of modified Grashof number $(\mathrm{Gc})$ on velocity. We observe that the velocity decreases with the increase of modified Grashof number (Gc)

with take the reverse action. Fig 5 illustrates the effect of porous parameter $(\mathrm{K})$ on velocity. We observe that the velocity decreases with the increase of porous parameter $(\mathrm{K})$ with take the reverse action. Fig 6 illustrates the effect of magnetic field (M) on velocity. We observe that the velocity decreases with the increase of magnetic field (M) with take the reverse action. Fig 7 illustrates the effect of radiation parameter $(\mathrm{R})$ on velocity. We observe that the velocity decreases with the increase of radiation parameter (R). Fig 8 shows the temperature for different values of heat generation (B). It is observe that the temperature increases with the increase of heat generation (B). From fig 9 shows the temperature for different values of Casson parameter $(\beta)$. We observe that the temperature increases with the increase of Casson parameter $(\beta)$. Fig 10 shows the temperature for different values of chemical reaction parameter $(\mathrm{Ch})$. It is seen that the temperature decreases with the increase of chemical reaction parameter (Ch).Fig 11 shows the temperature for different values of modified Grashof number (Gc). It is seen that the temperature decreases with the increase of modified Grashof number (Gc). Fig 12 shows the temperature for different values of Grashof number (Gr). It is seen that the temperature increases with the increase of Grashof number (Gc). Fig 13 shows the temperature for different values of porous parameter $(\mathrm{K})$. It is seen that the temperature decreases with the increase of porous parameter $(\mathrm{K})$. Fig 15 shows the temperature for different values of Prandtl number (Pr). It is seen that the temperature decreases with the increase of modified Prandtl number (Pr).Fig 15 shows the temperature for different values of radiation parameter $(\mathrm{Ra})$. It is seen that the temperature decreases with the increase of radiation parameter $(\mathrm{Ra})$. Fig 16 shows the concentration for different values of heat generation (B). It is seen that the temperature decreases with the increase of heat generation (B).Fig 17 shows the 
concentration for different values of Casson parameter $(\beta)$. It is seen that the concentration increases with the increase of Casson parameter ( $\beta$ ).Fig 18 shows the concentration for different values of chemical reaction (Ch). It is seen that the concentration decreases with the increase of chemical reaction (Gc).Fig 19 shows the concentration for different values of modified Grashof number $(\mathrm{Gc})$. It is seen that the temperature decreases with the increase of modified Grashof number (Gc).Fig 20 shows the concentration for different values of Grashof number (Gr). It is seen that the concentration increases with the increase of Grashof number (Gr).Fig 21 shows the concentration for different values of porous

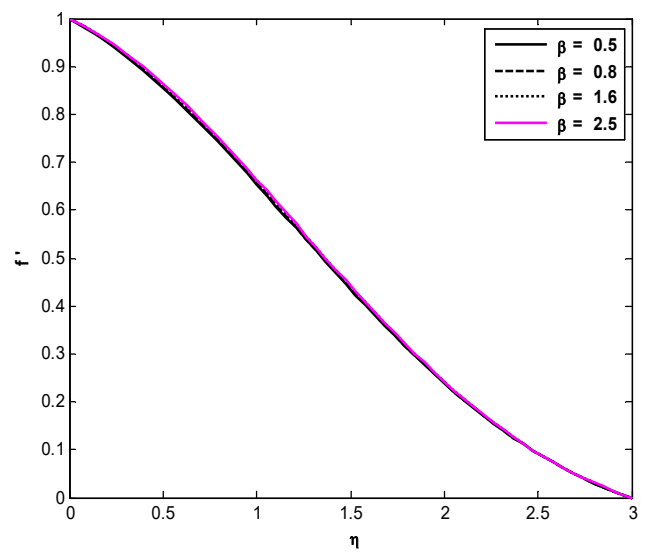

\section{Fig 1: The Velocity Profile for different values for Casson Parameter ( $\beta$ )}

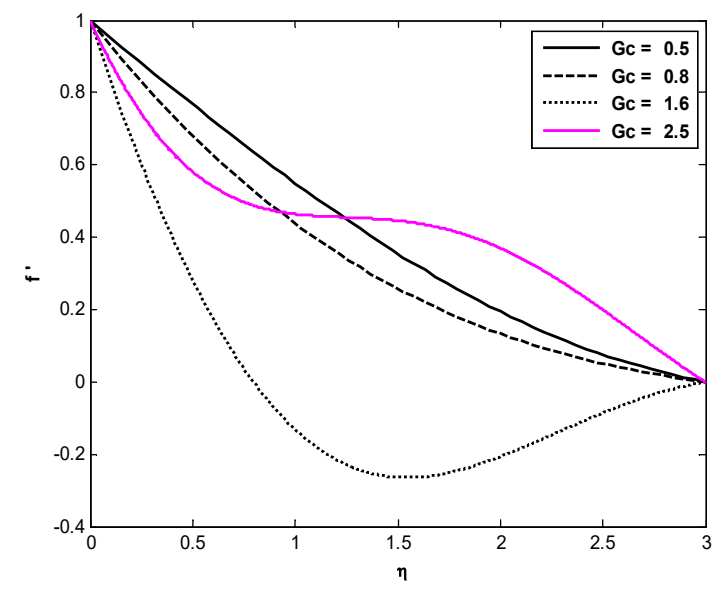

Fig 3: The velocity profile for the different values for modified Grashof number (Gc) parameter (K). It is seen that the concentrationincreases with the increase of porous parameter (K).Fig 22 shows the concentration for different values of magnetic parameter (M). It is seen that the concentration increases with the increase of magnetic parameter (M).Fig 23 shows the concentration for different values of Schmitt number (Sc). It is seen that the concentrationdecreases with the increase of Schmitt number (Sc).Fig 24 shows the concentration for different values of Soret number (Sr). It is seen that the concentration decreases with the increase of Soret number ( $\mathrm{Sr}$ ).

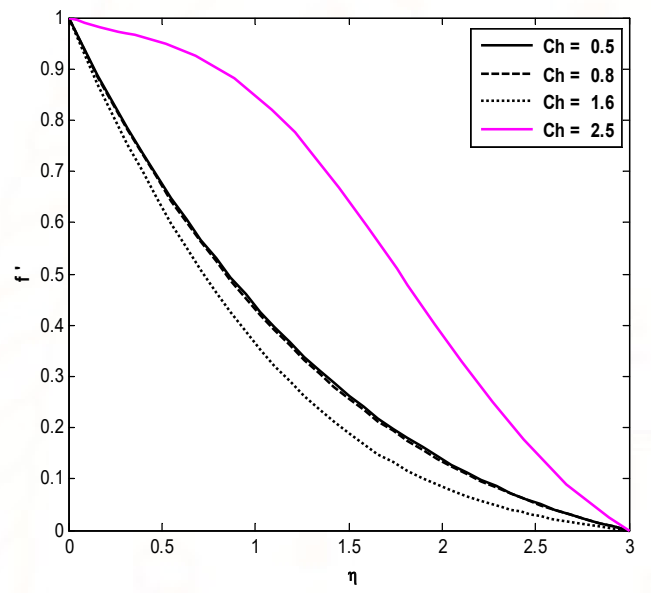

Fig 2: The velocity profile for the different values for chemical reaction $(\mathrm{Ch})$

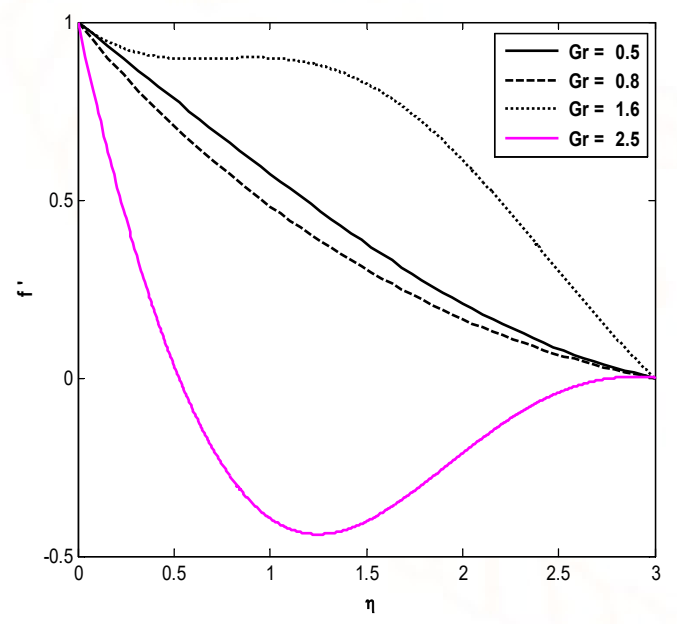

Fig 4: The velocity profile for the different values for Grashof number (Gr) 


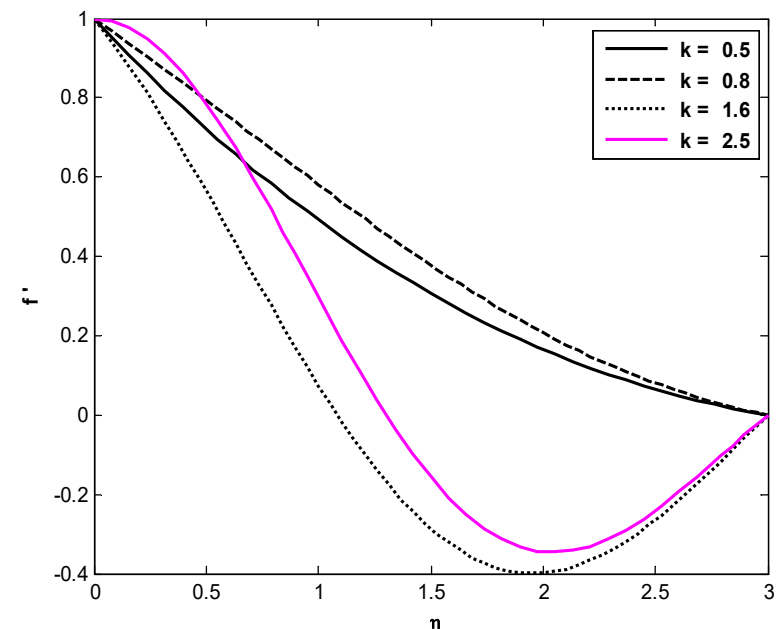

Fig 5: The velocity profile for the different values for porous parameter $(\mathrm{K})$

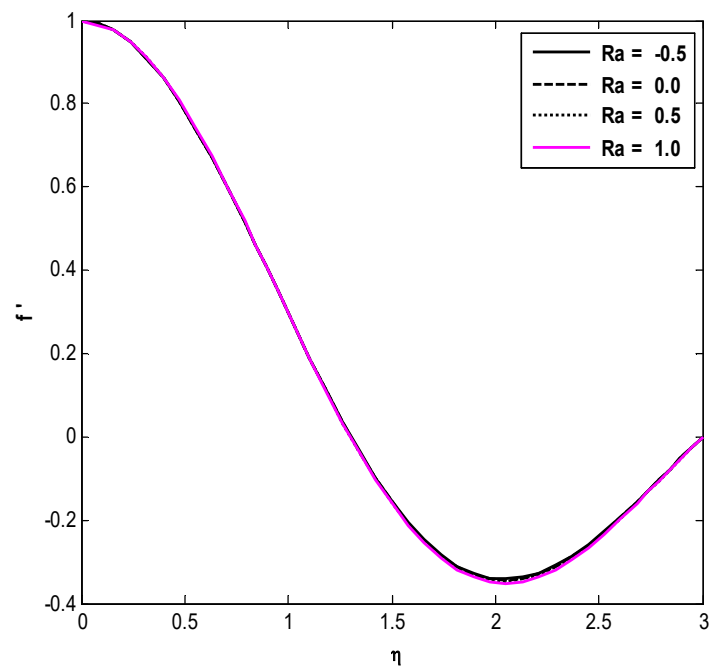

Fig 7: The velocity profile for the different values for radiation parameter $(R)$

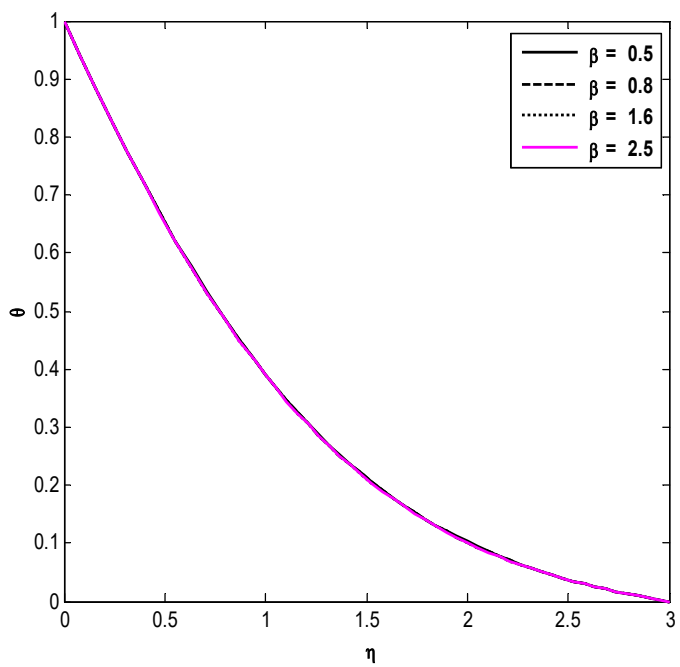

Fig 9: The temperature profile for the different values for Casson parameter $(\beta)$

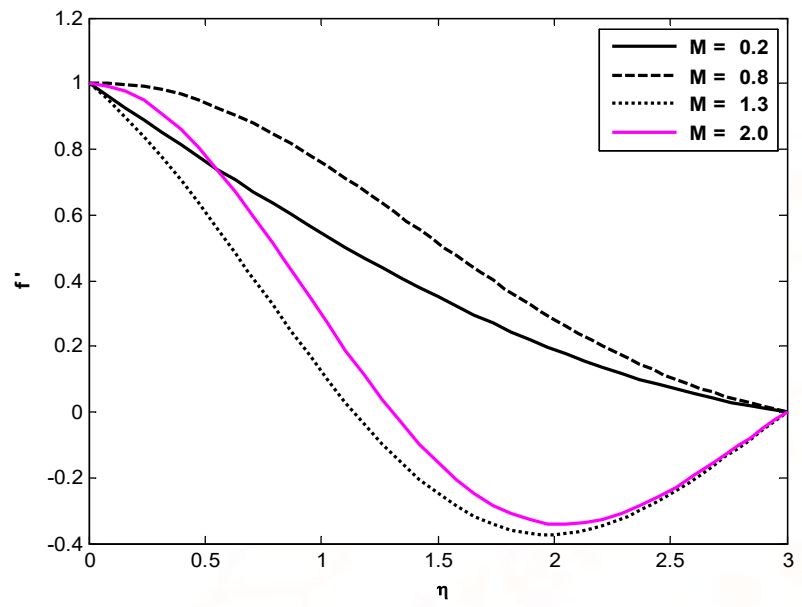

Fig 6: The velocity profile for the different values for magnetic field (M)

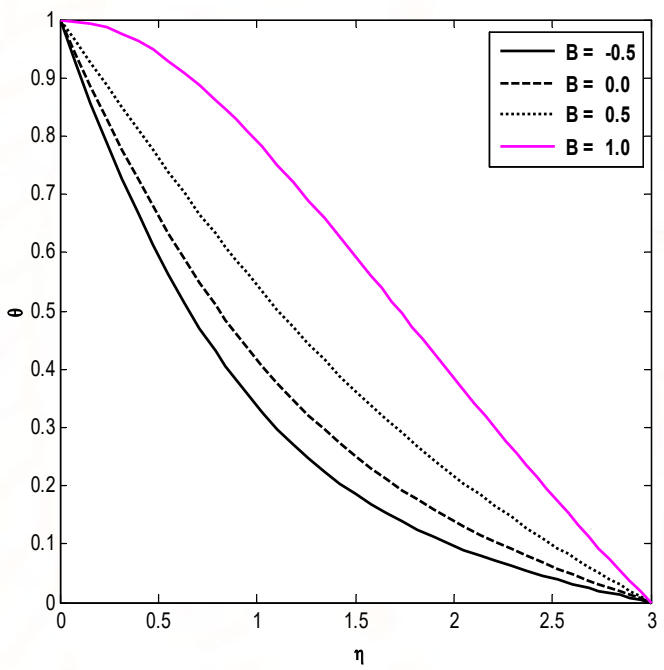

Fig 8: The temperature profile for the different values for heat generation (B)

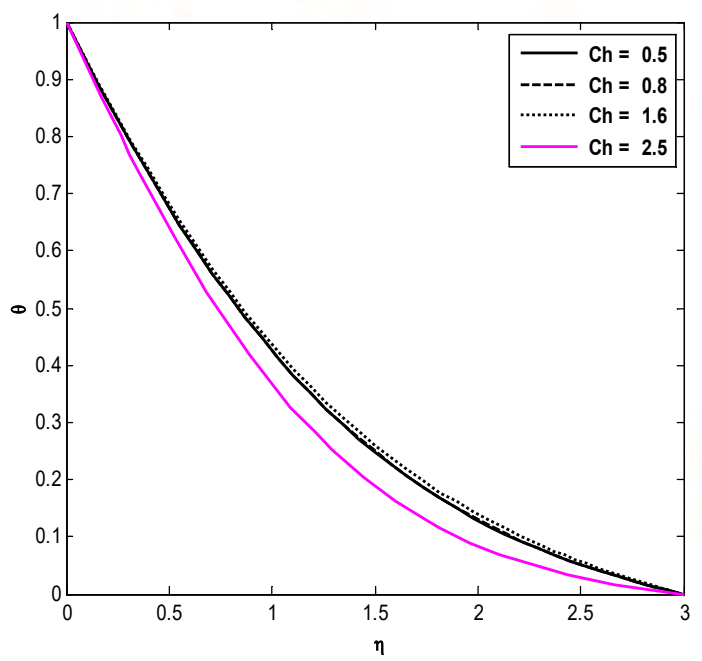

Fig 10: The temperature profile for the different values for Chemical Reaction (Ch) 


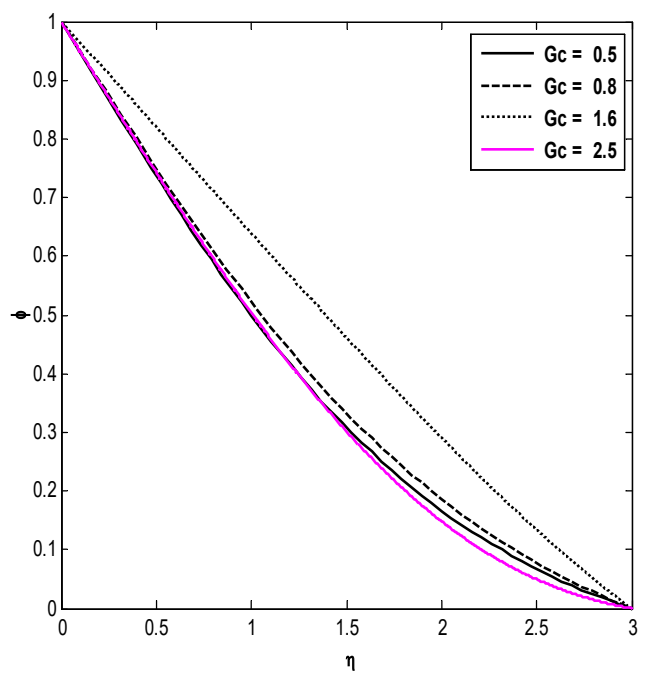

Fig 11: The temperature profile for the different values for modified Grashof number (Gc)

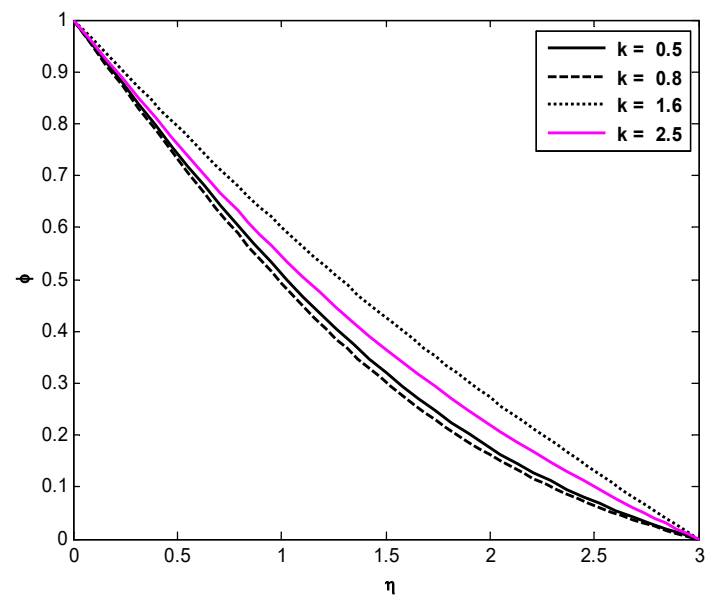

Fig 13: The temperature profile for the different values for porous parameter $(\mathbf{K})$

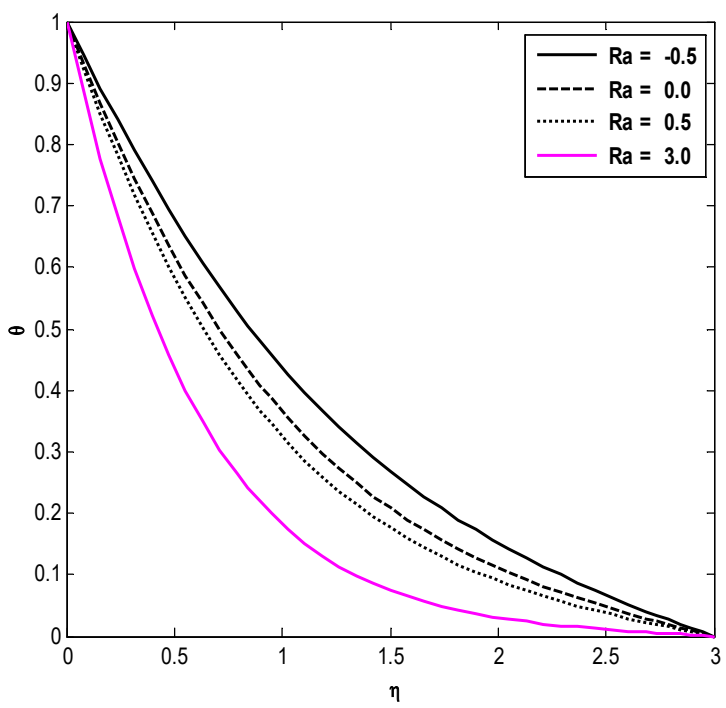

Fig 15: The temperature profile for the different values for radiation parameter $(\mathrm{Ra})$

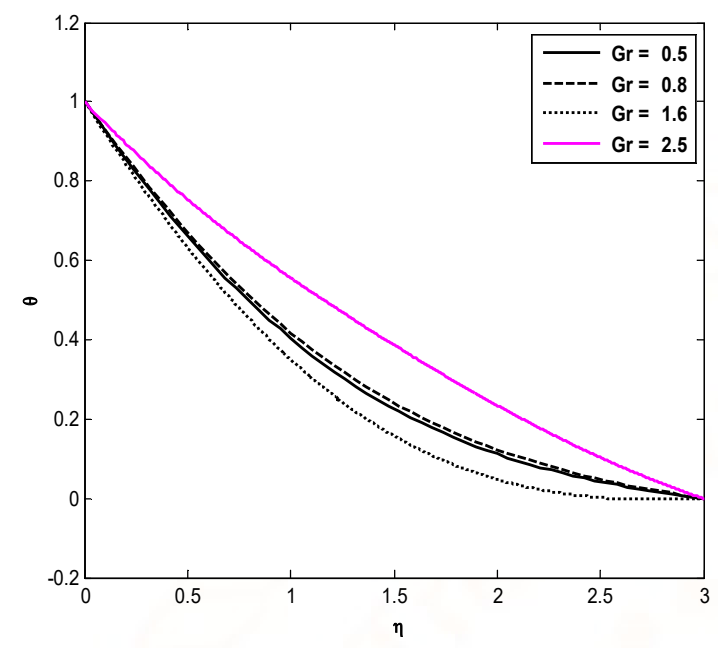

Fig 12: The temperature profile for the different values for Grashof number (Gr)

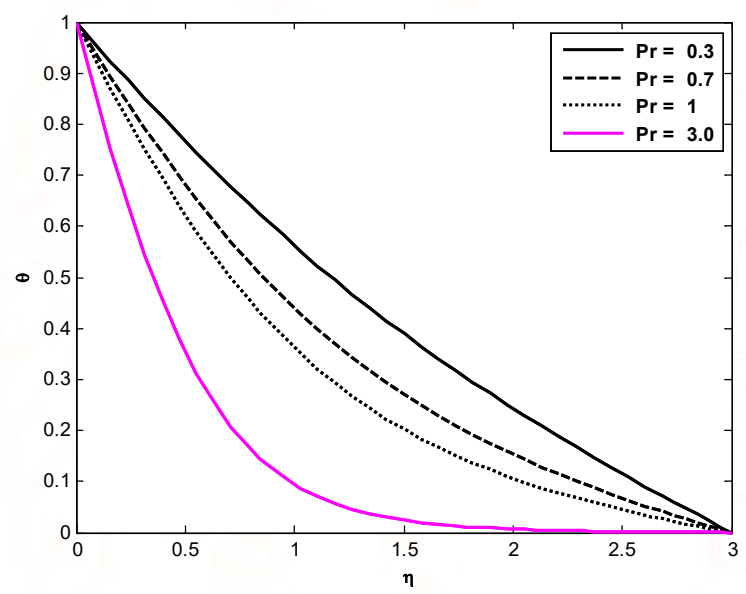

Fig 14: The temperature profile for the different values for Prandtl number (Pr)

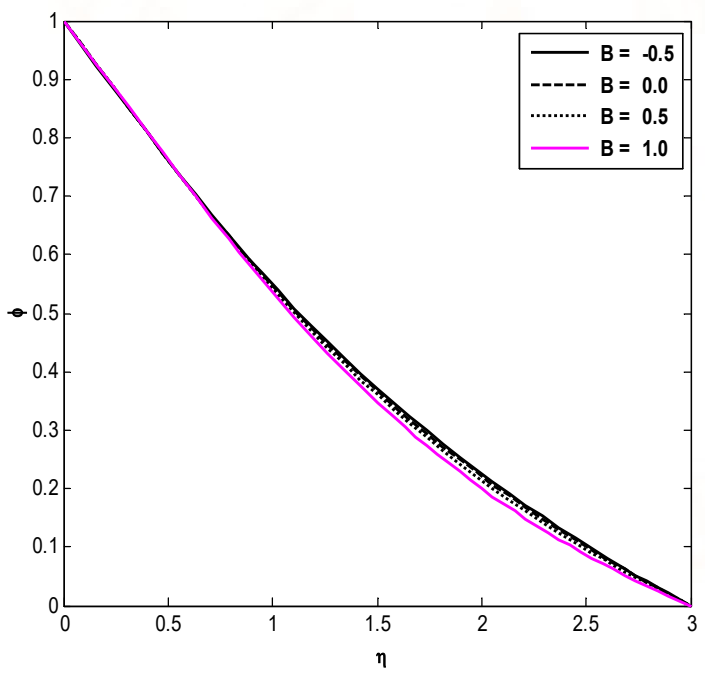

Fig 16: The Concentration profile for the different values for heat generation (B) 


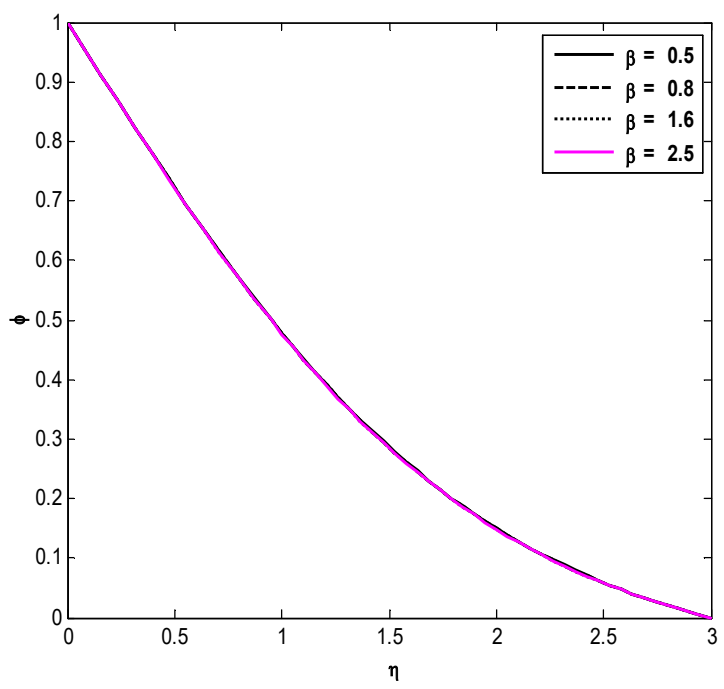

Fig 17: The Concentration profile for the different values for Casson parameter $(\boldsymbol{\beta})$

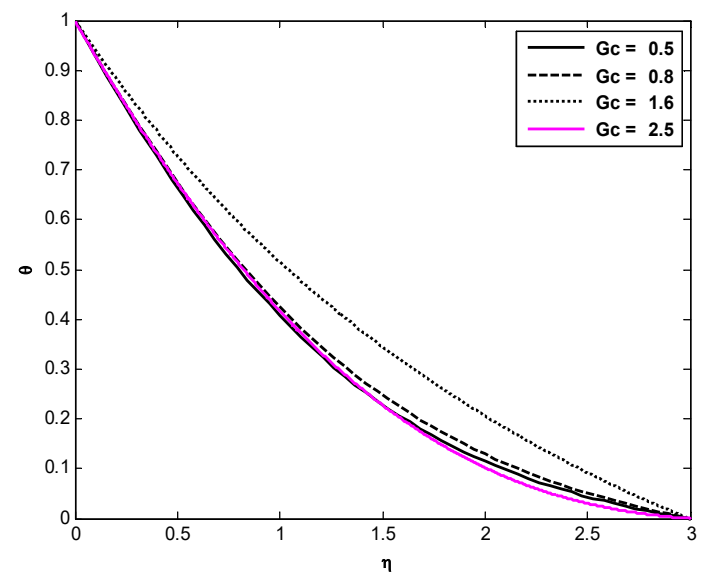

Fig 19: The Concentration profile for the different values for modified Grashof number (Gc)

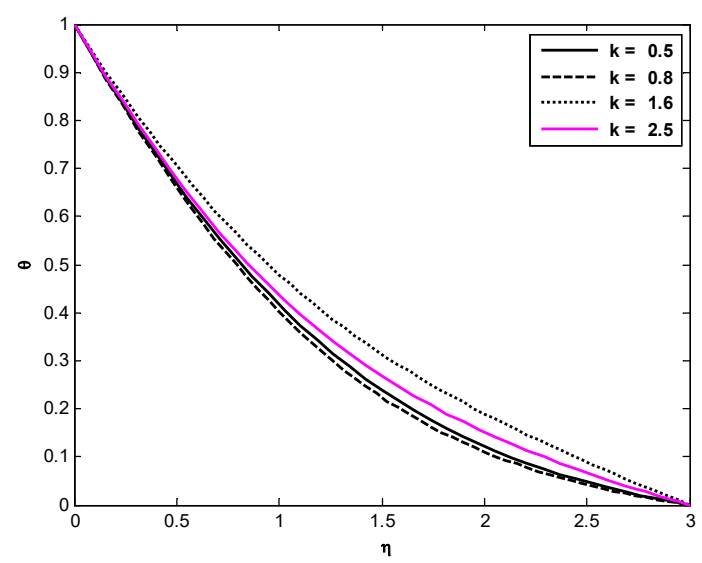

Fig 21: The Concentration profile for the different values for porous parameter $(\mathbf{K})$

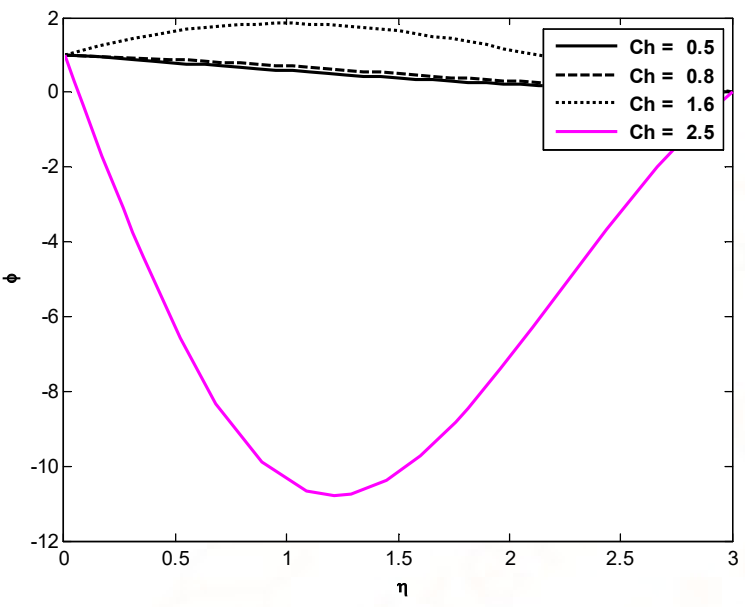

Fig 18: The Concentration profile for the different values for Chemical reaction $(\mathrm{Ch})$

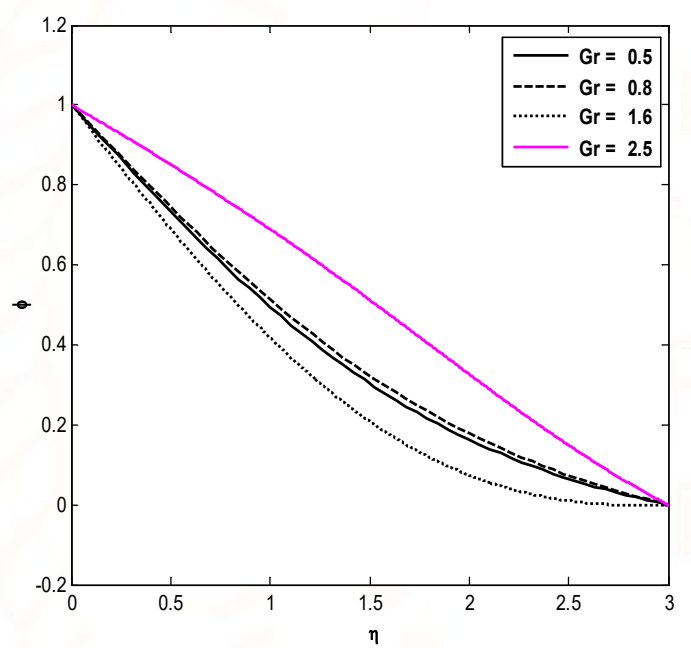

Fig 20: The Concentration profile for the different values for Grashof number (Gr)

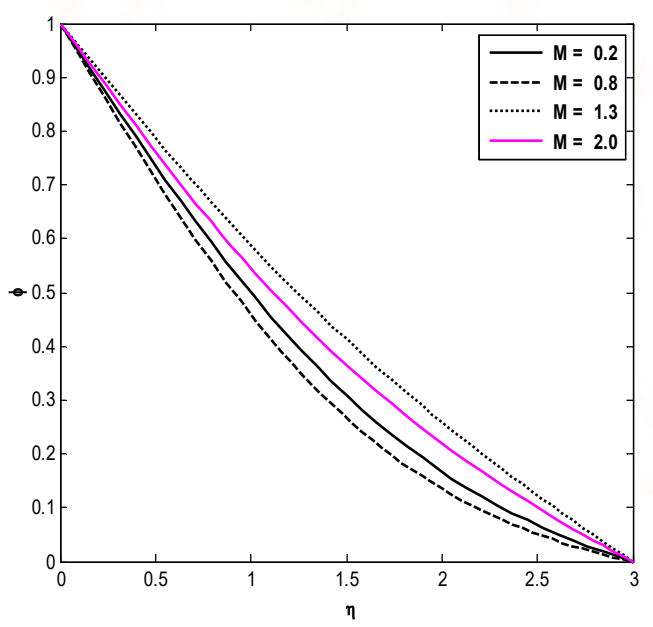

Fig 22: The Concentration profile for the different values for magnetic parameter (M) 


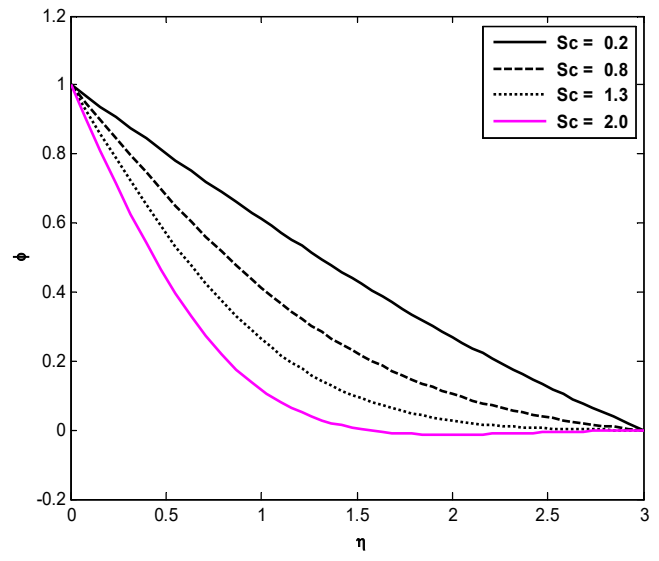

Fig 23: The Concentration profile for the different values for Schmitt number (Sc)

\section{CONCLUSIONS:}

This paper gives the effect of Heat and mass transfer on MHD boundary layer flow for a Casson fluid over a stretching sheet in presence of heat generation and chemical reaction. The governing equations are solved by shooting technique. It is observed that velocity is decreasing function of Casson parameter $\beta$, Magnetic parameter $M$, Suction parameter $S$. The thermal boundary layer thickness decreases with an increase in Prandtl number Pr. The effect of $\beta$ and $M$ on Skin friction coefficient is opposite.

We observe that the velocity decreases with the increase of Grashof number (Gr) with take the reverse action.

$>$ We observe that the velocity decreases with the increase of modified Grashof number (Gc) with take the reverse action.

$>$ It is observe that the temperature increases with the increase of heat generation (B).

$>$ We observe that the temperature increases with the increase of Casson parameter $(\beta)$.

$>$ It is seen that the temperature decreases with the increase of heat generation (B).

$>$ It is seen that the temperature decreases with the increase of modified Grashof number (Gc).

$>$ It is seen that the concentration increases with the increase of Grashof number (Gr).

$>$ It is seen that the concentration decreases with the increase of Soret number $(\mathrm{Sr})$.

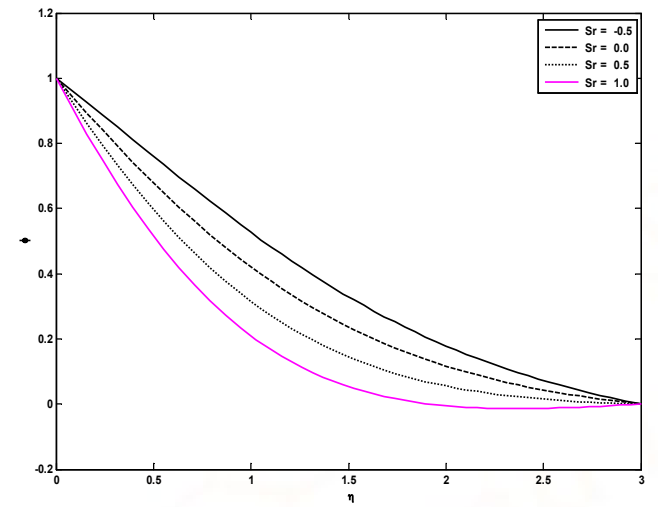

Fig 24: The Concentration profile for the different values for Soret effect (Sr)

\section{REFERENCES}

1. Abel S., Prasad K. V, and Mahaboob A., "Buoyancy force and thermal radiation effects in MHD boundary layer visco-elastic fluid flow over continuously moving stretching surface", International Journal of Thermal Sciences, vol. 44, pp. 465-476, 2005.

2. Ahmed A.A., "Similarity solution in MHD: Effects of thermal diffusion and diffusion thermo on free convective heat and mass transfer over a stretching surface considering suction or injection", Commun Nonlinear SciNumerSimulat, vol. 14, pp 2202-2214, 2009.

3. Andersson H. I., "MHD flow of a viscoelastic fluid past a stretching surface," ActaMechanica, vol. 95, pp. 227-230, 1992.

4. Anjalidevi S. P. and Kandasamy R., "Effects of chemical reaction, heat and mass transfer on laminar flow along a semi infinite horizontal plate", Heat andMass Transfer, vol. 35, pp. 465467, 1999.

5. Chen $\mathrm{CH}$., "Combined heat and mass transfer in MHD free convection from a vertical surface with Ohmic heating and viscous dissipation", Int J EngSci, vol. 42, pp. 699-713, 2004.

6. Crane L. J., "Flow past a stretching plate", Zeitschriftf urAngewandteMathematikundPhysik, vol. 21, pp. 645-647, 1970.

7. Das U. N., Deka R., and Soundalgekar V. M., "Effects of mass transfer on flowpast an impulsively started infinite vertical plate with constant heat flux and chemical reaction", ForschungimIngenieurwesen / Engineering Research, vol. 60, pp. 284 - 287, 1994.

8. Dash. R. K, Jayaraman G. and Mehta. K. N, 
"Shear-augmented dispersion of a solute in a Casson fluid flowing in a conduit", Annals of Biomedical Engineering., vol. 28, pp. 373-385, 2000.

9. Elbashbeshy E.M.A., "Heat and mass transfer along a vertical plate with variable surface tension and concentration in the presence of the magnetic field", Int J EngSci, vol. 35, pp. 515-22, 1997.

10. Eldabe N.T.M. and Salwa M.G.E. "Heat transfer of MHD non-Newtonian Casson fluid flow between two rotating cylinders", J. Phys. Soc. Japan, Vol. 64, pp. 4164, 1995.

11. Hayat, T., Shehzad, S.A., alsaedi, A., alhothuali, M.S., "Mixed Convection Stagnation Point Flow of Casson Fluid with Convective Boundary Conditions," Chin. Phys. Lett., vol. 29, pp. 114704 , 2012.

12. Attia H.A., Mohamed Eissa Sayed-Ahmed, "Transient mhdcouette flow of a casson fluid between parallel plates with heat transfer", Italian journal of pure and applied mathematics , pp. 19- 38, 2010.

13. Hossain M.A., Mandal A.C., "Mass transfer effects on the unsteady hydromagnetic free convection flow past an accelerated vertical porous plate", J Phys D: ApplPhys, vol. 18, 163-169, 1985.

14. Jha B.K., "MHD free convection and mass transform flow through a porous medium", Astrophys Space Sci, vol. 175, pp. 283-9, 1991.

15. Makinde O. D., "Free-convection flow with thermal radiation and mass transfer past a moving vertical porous plate", Int. Commun. Heat Mass Transfer, vol. 32, pp. 1411-1419, 2005.

16. McDonald D. A., Blood Flows in Arteries, 2nd Edition, Arnold, London, 1974

17. Merril E. W., Benis A. M., Gilliland E. R., Sherwood T. K., Salzman E. W., J. Appl.Physiol., vol. 20, pp. 954-967, 1965.

18. Mukhopadhyay S. and Layek G. C., "Effectsof thermal radiation and variable fluid viscosity on free convective flow and heat transfer past a porous stretching surface", International Journal of Heat and Mass Transfer, vol. 51, pp. 2167-2178, 2008.

19. Mustafa M., Hayat T., Pop I. and Aziz A. ' Unsteady boundary layer flow of a Casson fluid due to an impulsively started moving flat plate', Heat Transfer Asian Res., vol. 40, pp. 563-576 (2011).

20. Mustafa M., Hayat T., Pop I. and Hendi A. A., "Stagnation-point flow and heat transfer of a Casson fluid towards a stretching sheet", Z. Naturforsch., vol. 67, pp.70, 2012.

21. Mustafa M., Hayat T., Pop I. and Aziz A., "Unsteady boundary layer flow of a Casson fluid due to an impulsively started moving flat plate", Heat Transfer-Asian Resc, vol. 40, 563-76, 2011.

22. Muthucumaraswamy R. and Chandrakala P., "Radiative heat and mass transfer effect on moving isothermal vertical plate in the presence of chemical reaction", International Journal of Applied Mechanics and Engineering, vol.11, pp.639-646, 2006.

23. Nagarani P., Sarojamma G., Jayaraman G., "Effect of boundary absorption on dispersion in Casson fluid flow in an annulus: application to catheterized artery", ActaMechanica, vol 202, pp 47-63, 2009.

24. Nagarani P., Sarojamma G. and Jayaraman G, 2004, "Effect of boundary absorption in dispersion in Casson fluid flow in a tube", Ann Biomed Eng., pp. 706-19, 2004.

25. Nakamura M. and Sawada T., "Numerical Study on the Flow of a Non-Newtonian Fluid Through an axisymmetric Stenosis," ASME J. Biomechanical Eng., vol. 110, pp. 137-143, 1988.

26. Pal D., "Heat and mass transfer in stagnationpoint flow towards a stretching surface in the presence of buoyancy force and thermal radiation", Meccanica, vol. 44, pp. 145-158, 2009.

27. Postelnicu, "Influence of a magnetic field on heat and mass transfer by natural convection from vertical surfaces in porous media considering Soret and Dufour effects", Int. J. Heat Mass Transfer, vol. 47, pp. 1467-1472, 2004.

28. Rajagopal K. R., Na T. Y., and Gupta A. S., "Flow of a viscoelastic fluid over a stretching sheet", RheologicaActa, vol. 23, pp. 213-215, 1984.

29. Sarojamma. G, Ramana. B and Vendabai. K, "Heat and Mass transfer on MHD boundary layer flow of a chemically reacting non-Newtonian flow over a Stretching sheet with suction" International Journal of Engineering Sciences and research technology, Vol. 3(5), pp. 197-205, 2014.

30. Shehzad S. A., Hayat T., Qasim M. and Asghar S., "Effects of mass transfer on MHD flow of Casson fluid with chemical reaction and suction", Brazilian Journal of Chemical Engineering, Vol. 30, pp. 187 - 195, 2013. 